\title{
Association between non-coding circular RNAs and papillary thyroid carcinoma (Review)
}

\author{
NEGIN SOGHLI ${ }^{1}$, TOOBA YOUSEFI ${ }^{2,3}$ and DURDI QUJEQ ${ }^{2,4,5}$ \\ ${ }^{1}$ Dental Materials Research Center, Institute of Health, Babol University of Medical Sciences; \\ ${ }^{2}$ Department of Clinical Biochemistry, School of Medicine, Babol University of Medical Sciences; \\ ${ }^{3}$ Student Research Committee, Babol University of Medical Sciences; ${ }^{4}$ Cellular and Molecular Biology \\ Research Center (CMBRC), Health Research Institute, Babol University of Medical Sciences; ${ }^{5}$ Cancer Research Center, \\ Health Research Institute, Babol University of Medical Sciences, Babol 47176-47745, Iran
}

Received February 19, 2020; Accepted May 8, 2020

DOI:10.3892/wasj.2020.51

\begin{abstract}
Papillary thyroid carcinoma (PTC) accounts for $>80 \%$ of thyroid cancer cases with an increasing incidence worldwide. Despite improved diagnostic strategies and treatments, certain aggressive characteristics of PTC render the identification of alternative therapeutic approaches necessary. Circular RNAs (circRNAs) are a novel class of long non-coding RNAs (lncRNAs) containing a closed-loop structure with a covalent linkage of $3^{\prime}-5$ ' ends. Thus, they are more stable and resistant to RNA exonucleases. A significant number of recent studies on circRNAs have illustrated the dysregulated expression of these biomarkers in various types of tumors. However, the function of these non-coding RNAs and downstream mechanisms in PTC have not yet been entirely identified. In the present review article, the authors aim to discuss and summarize the functions and roles of circRNAs, including their expression profiles, potential targets and regulatory mechanisms previously recognized in PTC.
\end{abstract}

\section{Contents}

1. Introduction

2. Roles of circRNAs in PTC cell progression

3. Conclusion

Correspondence to: Professor Durdi Qujeq, Department of Clinical Biochemistry, School of Medicine, Babol University of Medical Sciences, 1 Ganjafrooze Street, Babol 47176-47745, Iran

E-mail: dqujeq@gmail.com; d.qujeq@mubabol.ac.ir

Abbreviations: circRNAs, circular RNAs; ncRNAs, non-coding RNAs; miRNAs or miRs, microRNAs; lncRNAs, long non-coding RNAs; PTC, papillary thyroid carcinoma

Key words: non-coding circular RNA, papillary thyroid carcinoma

\section{Introduction}

Papillary thyroid carcinoma (PTC). Thyroid cancer, originating from thyroid follicular epithelial cells, is the most common type of cancer of the endocrine system, exhibiting a rapid increase in mortality rate worldwide (1-3). There are 4 subtypes of thyroid cancer, including papillary thyroid cancer (PTC), follicular thyroid cancer (FTC), anaplastic thyroid cancer (ATC) and medullary thyroid cancer (MTC) (4). PTC accounts for $>80 \%$ of all thyroid cancer cases $(1,5,6)$. Among all types of thyroid cancers, the incidence of PTC is much higher and is often observed mainly among younger patients (7). Genetic mutations and environmental exposure are considered risk factors of PTC (8). The optimal treatment period for PTC is the early stages of the disease; this period is often surpassed by the time of diagnosis, due to the slow growth rate and atypical symptoms (9). Despite the increase in the 5-year survival rate up to $90 \%$ and favorable prognosis in the majority of patients in the case of prompt treatment, including thyroidectomy and adjuvant radioactive iodine therapy, certain aggressive phenotypes of PTC, such as extra-thyroidal extension, multifocal tumors, and lymph node and distant metastases lead to a poor prognosis $(10,11)$. Hence, it is of utmost importance to identify novel diagnostic and therapeutic targets for PTC.

It has recently been demonstrated that genetic factors affect thyroid cancer progression (12). Various molecular deregulations direct the tumorigenicity of PTC. BRAFV600E is known as a highly specific prognostic factor for patients with PTC. It functions by activating mitogen-activated protein kinase (MAPK) that has previously been proven to participate in various human cancers (13-15). Although the diagnostic strategies for thyroid cancer have greatly improved, fine needle aspiration (FNA) cytology remains the gold standard technique, which has a success rate of $70 \%$ in determining the findings $(16,17)$. Therefore, it is vital to identify novel diagnostic, prognostic and therapeutic biomarkers in order to diagnose thyroid cancer during the early stages.

Characteristics and roles of circular RNAs (circRNAs) General characteristics. Non-coding RNAs (ncRNAs) form the majority of the human transcriptome. A number 
of regulatory mechanisms and pathophysiological pathways are controlled by these ncRNAs, including microRNAs (miRNAs or miRs), long non-coding RNAs (lncRNAs) and circRNAs (18). The existence of circRNAs in human cells was first observed and reported by Kos et al in 1986 (19). Recently, more investigations have been conducted on the function of circRNAs in various diseases and circRNAs have become a hotspot in cancer research. circRNAs are a product of a back-splicing mechanism containing a single-stranded covalently closed-loop structure possessing neither 5'-3' polarity nor a polyadenylated tail $(18,20)$. These characters render them more stable and resistant to RNase R (21). circRNAs are mainly located in the cytoplasm and are found in a wide range of living organisms (22). Given their interesting features, the present review article aimed to summarize their main functions in cancer.

circRNAs as miRNA sponges. Predominantly, circRNAs act as miRNA sponges and regulate the cell progression and cell cycle by modulating gene expression or protein-generation in transcriptional or post-transcriptional stages (23). Similar to other ncRNAs, circRNAs have been found to play a pivotal role in the onset of diseases $(24,25)$. Recent studies have demonstrated that a number of circRNAs function as tumor-promoters or tumor-suppressors. For instance, the overexpression of circCDR1as in osteosarcoma cells sponges miR-7 and suppresses the inhibitory effects of miR-7 on osteosarcoma cell progression $(26,27)$. In addition, the upregulation of circRNA HIPK3 has been shown to promote the development of gall bladder cancer by sponging miR-124 and acting as a tumor-promoter (28).

Role of circRNAs in cancer. miRNAs have previously been used as molecular biomarkers in various types of cancer (29). Currently, researchers have found that the up- or downregulation of certain circRNAs can affect tumor progression in some diseases (30-32). However, the investigations on the functions of circRNA in cancerous tissues are insufficient, and their reliability for being used as diagnostic, prognostic or therapeutic biomarkers is indeterminate. circRNAs are resistant to exonucleases and RNase $\mathrm{R}$, and these characters render them more stable than other types of ncRNAs $(21,33)$. Hence, circRNAs may be acknowledged as ideal biomarkers for the diagnosis of cancer tissues.

\section{Roles of circRNAs in PTC cell progression.}

Microarray analysis has indicated that certain circRNAs are differently expressed between PTC and adjacent normal tissues; however, their function and downstream mechanisms remain largely unknown (34). A graphical abstract of the identified circRNAs that are dysregulated in PTC cells and their downstream signaling pathways is depicted in Fig. 1. Conducted studies on the role of circRNAs in PTC are limited; however, circRNAs have a more stable structure, they are considered as ideal biomarkers for diagnosis (33). In the present review, the deregulated circRNAs in PTC are discussed, with particular focus on their regulatory mechanisms and functions.

\section{Upregulated circRNAs in PTC}

hsa-circ-0058124. Conducting RT-qPCR, hsa-circ-0058124, located on chromosome 2 and generated from the fibronectin 1 gene $(35,36)$, has been demonstrated to be upregulated in PTC tissues and cell lines. Cell fraction assay and FISH have exhibited that this circRNA is mainly available in the nucleus and sometimes in the cell cytoplasm (37). The overexpression of hsa-circ-0058124 has been shown to result in the poor prognosis of patients with PTC; in addition, a larger tumor size, advanced stage, extra-thyroidal extension, lymph node metastasis and distant metastasis are more often observed in patients with a higher expression of hsa-circ-0058124. Western blot analysis and dual-luciferase assay have revealed that hsa-circ-0058124 upregulates NUMB expression by sponging miR-218-5p to suppress the NOTCH3 signaling pathway. hsa-circ-0058124 functions by restraining the NOTCH3/GATAD2A cascade (37). These findings suggest that hsa-circ-0058124 regulates PTC progression and functions as an oncogene; thus, it can be used as an ideal prognostic biomarker for patients with PTC.

hsa-circ-0039411. The overexpression of hsa-circ-0039411, located on chromosome 16 , has been proven by conducting RT-qPCR in PTC tissues and cell lines (38). Gain- and loss-of-function and CCK- 8 assays have demonstrated that hsa-circ-0039411 is positively associated with cell growth. Flow cytometric analysis have also shown that the downregulation of hsa-circ-0039411 leads to PTC cell apoptosis. Transwell assay has also confirmed that PTC cell migration and invasion are positively controlled by this biomarker (39). RT-qPCR, along with dual-luciferase assay, have revealed that hsa-circ-0039411 sponges miR-1179 and miR-1205, and two potential binding sites for miR-1205 have been recognized. hsa-circ-0039411 upregulates the expression of ABCA9 and MTA1 by sponging miR-1179 and miR-1205, respectively, and thus performs an oncogenic role in PTC cell progression (39). It can thus be concluded that hsa-circ-0039411 may be used as a therapeutic target in patients with PTC.

circBACH2. Cai et al have reported that $\operatorname{circBACH} 2$, also known as hsa-circ-0001627 and derived from exon 2 of chromosome 6, is upregulated in PTC tissues and cell lines. Previously, ROC curve analysis determined that circBACH2 expression level was upregulated in PTC tissues in comparison with adjacent non-tumor tissues; a longer survival lifetime was recognized among patients with higher expression levels of circBACH 2 by conducting Kaplan-Meier analysis (40). Previously, luciferase reporter assay also proved that miR-139-5p had some potential binding sites on circBACH2, and this circRNA functioned by sponging miR-139-5p. RT-qPCR, western blot analysis and CCK-8 assay revealed that circBACH2 sponged miR-139-5p and suppressed its inhibitory effect on LIM domain only 4 (LMO4) (40). These results indicate that circBACH2 mediates PTC cell progression and invasion by regulating the miR-139-5p/LMO4 axis.

circFOXM1. circFOXM1, also known as circ-0025033, is a product of the FOXM1 gene and is mapped on chromosome 12 . In a previous study, by performing circRNA microarray analysis and RT-qPCR, it was found that the expression level of circFOXM1 was upregulated in PTC tissues and cell lines. RT-qPCR, CCK-8 assay and colony formation assay also revealed that circFOXM1 affected cell proliferation. Cell apoptosis and metastasis were also found to be controlled by circFOXM1, and the overexpression of this biomarker promoted PTC cell migration and invasion (38). In 



Figure 1. Upregulated and downregulated circRNAs in PTC. circRNAs, circular RNAs; PTC, papillary thyroid carcinoma.

previous studies, bioinformatics analysis also demonstrated that circFOXM1 functioned as a sponge for both miR-1231 and miR-1304. These two miRNAs were reported to function as tumor-suppressors (41-43). In a previous study by Pan et al, CCK-8 and Transwell assay conducted demonstrated that circFOXM1 negatively regulated the expression of miR-1231 and miR-1304, and played an oncogenic role in patients with PTC (38). These data indicate that circFOXM1 promotes PTC progression by sponging miR-1231 and miR-1304.

circUGGT2. Zhou et al described circUGGT2, also known as hsa-circ-0008274 and located on chromosome 11, as an upregulated biomarker in PTC tissues and cell lines (44). These data were obtained using bioinformatics analysis and RT-qPCR. In addition, in that same study, it was found out that the high expression of circUGGT2 was associated with TNM stage and lymph node metastasis, but not with age, sex, extra-thyroidal extension, primary tumor and tumor size. Furthermore, MTT assay was conducted and it was shown that circUGGT2 regulated PTC cell proliferation. In the same study, further investigations revealed that the invasion of PTC cells was contributed to circUGGT2. Western blot analysis was also performed to explore the downstream mechanisms. The results revealed that the overexpression of circUGGT2 led to the suppression of the 5'AMP-activated protein kinase (AMPK)/mammalian target of rapamycin (mTOR) signaling pathway (44). The AMPK/mTOR signaling pathway has been shown to inhibit autophagy and increase cell proliferation in both normal and tumor tissues (45). Taken together, it can be concluded that circUGGT2 mediates PTC cell progression and invasion by regulating the AMPK/mTOR signaling pathway.

circEIF6. In a previous study, bioinformatics and RT-qPCR confirmed that circEIF6 (hsa-circ-0060060) was upregulated in PTC tissues and cell lines. Conducting sequence analysis, two potential binding sites were assessed between circEIF6 and miR-144-3p. RT-qPCR also revealed that the level of miR-144-3p was decreased in PTC tissues and cell lines. Dual-luciferase reporter assay proved that circEIF6 sponged miR-144-3p and regulated the expression of TGF- $\alpha$ as a result in cisplatin-treated cells (46). Previous studies have reported miR-144-3p can function as either tumor-promoter or tumor-suppressor $(47,48)$. Previously, the results of MTT assay and flow cytometry demonstrated that the overexpression of circEIF6 enhanced cell proliferation and autophagy, and suppressed cell apoptosis (46). These data suggest that circEIF6 promotes PTC progression by activating the miR-144-3p/TGF- $\alpha$ signaling pathway.

circ-102171. circ-102171 has been shown to be significantly upregulated in PTC tissues and cell lines. In a previous study, functional assays, including CCK-8 and colony formation assays, demonstrated that circ-102171 regulated the proliferation and apoptosis of PTC cells, and affected their cell cycle. Performing Transwell assay indicated that the knockdown of circ-102171 suppressed the migration and invasion of PTC cells. Using silver staining, MS 


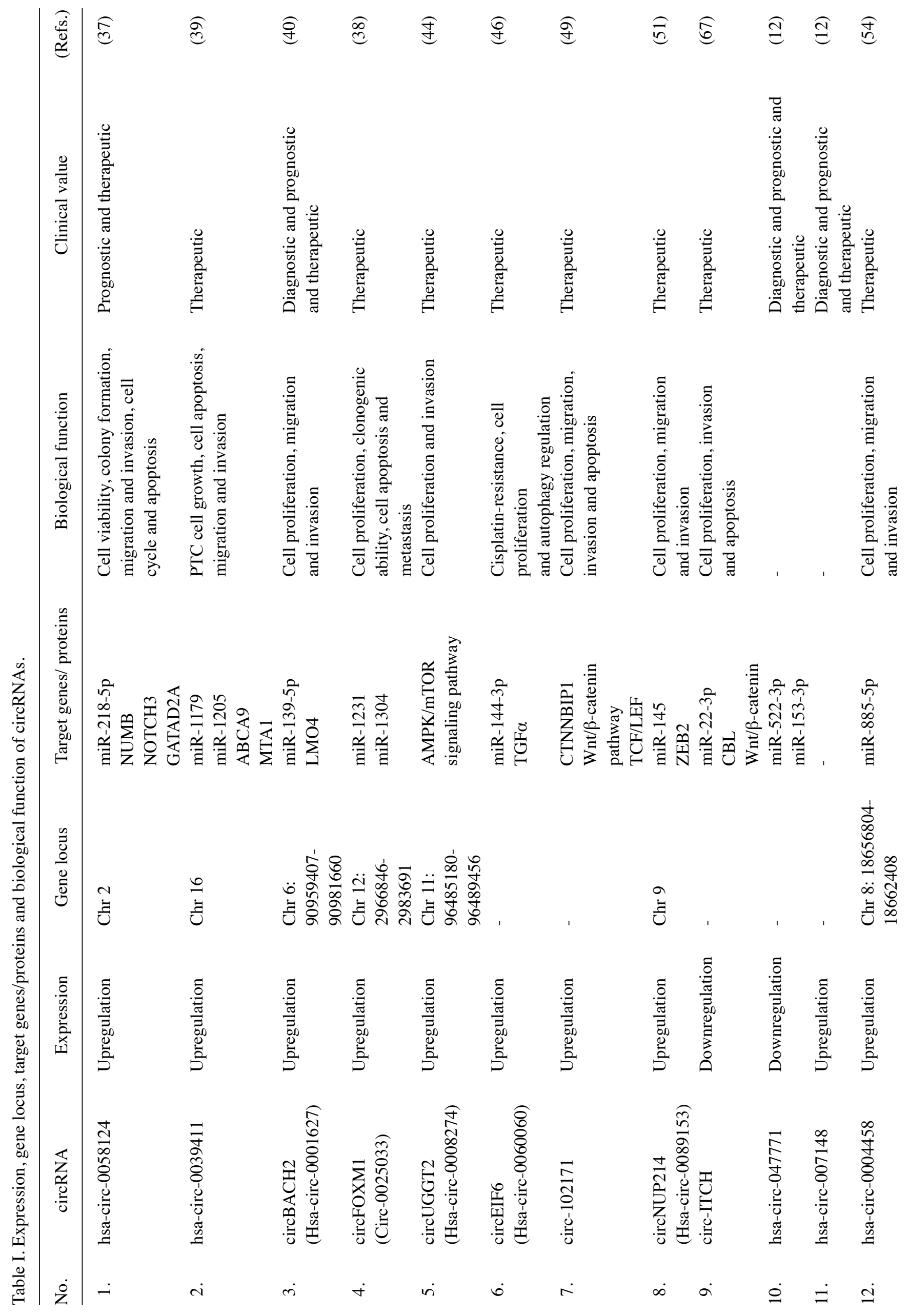


identification, biotin-labeled probes and RNA-EMSA assay, it was also found that circ-102171 interacted with CTNNBIP1 protein in PTC cells (49). CTNNBIP1 has been shown to be negatively associated with the $\mathrm{Wnt} / \beta$-catenin pathway by regulating the $\beta$-catenin-TCF/LEF cascade. As a result, the overexpression of circ-102171 leads to the increased activation of the Wnt/ $\beta$-catenin signaling pathway (50). In a previous study, RT-qPCR and functional experiments revealed that CTNNBIP1 was downregulated in PTC cells, and this protein functioned as a tumor-suppressor (49). To sum up, these data confirm that circ-102171 controls PTC cell progression and invasion by activating the $\mathrm{Wnt} / \beta$-catenin pathway by regulating CTNNBIP1.

circNUP214. circNUP214, also known as hsa-circ-0089153, is located on chromosome 9. In a previous study, bioinformatics and RT-qPCR revealed that this circRNA was significantly upregulated in PTC tissues and cell lines. Localized by fluorescence in situ hybridization, circNUP214 was found to be mainly located in the cytoplasm. CCK-8 and colony formation assays revealed that the expression of circNUP214 was directly associated with the proliferation and apoptosis of PTC cells. Transwell assays also demonstrated that the circNUP214 expression level was associated with PTC cell migration and invasion. By conducting the dual-luciferase assay, it was also confirmed that circNUP214 enhanced zinc finger E-box-binding homeobox 2 (ZEB2) expression by sponging miR-145 (51). ZEB2 has previously been reported to function as an oncogene in various tumor tissues $(52,53)$. These results suggest that the overexpression of circNUP214 promotes PTC cell proliferation and invasion by sponging miR-145 in a ZEB2-dependent manner.

hsa-circ-007148. In a previous study, bioinformatics and RT-qPCR validated that hsa-circ-007148 expression was enhanced in PTC tissues and cell lines. It was also found that the overexpression of hsa-circ-007148 was significantly associated with lymph node metastasis; however, no other clinicopathological association was detected. ROC curve analysis also indicated that hsa-circ-007148 may be used as a diagnostic biomarker for distinguishing PTC tissues (12). These data suggest that the upregulation of hsa-circ-007148 plays an oncogenic role in patients with PTC.

hsa-circ-0004458. hsa-circ-0004458, located on chromosome 8: 18656804-18662408 with 448 nucleotides in length, was first detected in gastric cancer and PTC. The upregulation of this biomarker was previously confirmed among PTC tissues and cell lines by performing RT-qPCR. It was also proven that hsa-circ-0004458 expression was associated with tumor size, invasion, lymphatic metastasis, distant metastasis and TNM stage. Following the knockdown of hsa-circ-0004458, a reduction in tumor growth was also observed, as well as cell cycle arrest. Dual-luciferase assay confirmed that hsa-circ-0004458 modulated RAC1 by sponging miR-885-5p (54). RAC1 is a critical protein in underlying mechanisms responsible for cell growth, migration and the activation of various protein kinases $(55,56)$. These findings indicate that the hsa-circ-0004458/miR-885-5p/RAC1 signaling pathway regulates PTC cell progression and invasion.

circZFR. circZFR, also known as hsa-circ-0072088 and mapped on chromosome $5 \mathrm{p} 13.3$, plays a role in various tumor-regulating mechanisms $(57,58)$. In a previous study, 
bioinformatics and RT-qPCR revealed the upregulated expression of this biomarker in PTC tissues and cell lines. Moreover, the overexpression of circZFR in patients with PTC exhibited a positive association with clinical severity, including TNM stage and distant metastasis. Kaplan-Meier analysis also indicated that a higher expression of circZFR was associated with a poor prognosis. The effects of circZFR on cell proliferation and invasion were also confirmed by performing CCK-8, colony formation and Transwell assays, respectively. To explore the underlying mechanisms, a dual-luciferase reporter assay was conducted and it was validated that circZFRmodulated C8orf4 expression by sponging miR-1261 (59). C8orf4 is known as thyroid cancer 1 (TC1) and has been previously shown to play an oncogenic role in other cancerous tissues $(60,61)$. In conclusion, these data illustrate that circZFR regulates PTC cell proliferation and invasion via the miR-1261/C8orf4 axis.

circRNA plasmacytoma variant translocation gene 1 (circPVT1). circPVT1, located on chromosome 8q24, has been reported to promote PTC progression through different pathways. Exploring the potential role of circPVT1 in PTC, it was found that circPVT1 was upregulated in PTC tissues. Further investigations revealed that the T stage, lymph node metastasis and survival status were associated with a higher expression of circPVT1. Still, no association was found with age, sex and the ATA risk of patients. In addition, the overexpression of circPVT1 promoted apoptosis and inhibited the migration and invasion of PTC cells. Luciferase reporter assay demonstrated that circPVT1 had putative binding sites for miR-126. RIP assay and RT-qPCR indicated that circPVT1 sponged miR-126 and a reduction in miR-126 level occurred by circPVT1 overexpression (62). It has previously been demonstrated that miR-126 functions as a tumor-suppressor (63). In summary, the upregulation of circPVT1 promotes the progression of PTC cells by sponging miR-126.

circRASSF2. hsa-circ-0059354, also known as circRASSF2 and derived from the RASSF2 gene on chromosome 20 : 4760668-4766974, was previously found to be upregulated in PTC tissues and cell lines. Microarray analysis indicated that circRASSF2 was overexpressed in PTC tumor tissues with a $>10$-fold change. Further investigations confirmed that the higher expression of circRASSF2 was associated with tumor stage and lymph node metastasis. Colony formation and Transwell assays demonstrated that the overexpression of circRASSF2 promoted cell proliferation, and enhanced the cell migratory and invasive capabilities. Dual-luciferase reporter assay confirmed that circRASSF2 functioned as a sponge for miR-1178. Functional experiments also revealed that Toll-like receptor (TLR)4 was a direct target of miR-1178 in PTC tissues (64). Thus, these data suggest that circRASSF2 sponges miR-1178 and regulates PTC cell proliferation and invasion by targeting TLR4.

circ-0005273. By conducting RT-qPCR, a previous study found that circ-0005273 was upregulated in PTC tissues and cell lines. circ-0005273 was found to be mainly located in the cell cytoplasm and the presence of this biomarker indicated a poor prognosis of patients with PTC. Functional experiments revealed that circ-0005273 promoted PTC tumor growth and progression. Further investigations indicated that there were potential binding sites on circ- 0005273 for miR-1138. CCK-8, colony formation and Transwell assays demonstrated that circ-0005273 sponged miR-1138 and suppressed its inhibitory effect on sex-determining region Y (SRY)-box 2 (SOX2) (65). Thus, circ-0005273 plays an oncogenic role in PTC cells by regulating the circ-0005273/miR-1138/ SOX2 axis.

\section{Downregulated circRNAs in PTC tissues}

hsa-circ-100395. In a previous study, microarray analysis revealed that hsa-circ-100395 was downregulated in PTC tissues and cell lines. The lower expression of this biomarker was validated by conducting RT-qPCR and bioinformatics analysis. Functional experiments indicated that hsa-circ-100395 was associated with miR-141-3p and miR-200a-3p in PTC tissues. These two miRNAs were overexpressed in PTC tissues as a result of a downregulation that occurred in hsa-circ-100395 expression. Downstream cancer-related genes were sponged by miR-141-3p and miR-200a-3p and this led to PTC cell progression. Accordingly, hsa-circ-100395 regulated PTC cell progression by modulating the hsa-circ-100395/miR-141-3p/miR-200a-3p axis (34).

circITCH. circITCH plays a vital role in a variety of downstream mechanisms involved in tumorigenesis (66). Wang et al reported a downregulation in the expression of this biomarker in PTC tissues and cell lines, validated by RT-qPCR. CircITCH expression was also shown to be positively associated with clinical stage and the lymph node metastasis of patients with PTC. CCK-8 and Transwell assays demonstrated that the higher expression of circITCH led to the inhibition of the proliferation and invasion of PTC cells. Luciferase reporter assays indicated that circITCH functioned by sponging miR-22-3p. Moreover, functional experiments revealed that miR-22-3p had 3 potential binding sites for CBL (67). As has been previously demonstrated, CBL regulates PTC progression by regulating the $\mathrm{Wnt} / \beta$-catenin pathway (68). To sum up, circITCH functions as a tumor-suppressor by regulating the circITCH/miR-22-3p/CBL/ $\beta$-catenin pathway, and regulating the proliferation and invasion of PTC cells.

hsa-circ-047771. In a previous study microarray and RT-qPCR analysis revealed that hsa-circ-047771 was downregulated in PTC tissues and cell lines (12). Further investigations illustrated that there was a significant association between hsa-circ-047771 expression and BRAFV600 mutation, lymph node metastasis and TNM stage, whereas no association was observed with other clinicopathological features (12). BRAFV600 mutation has been used as a poor prognostic marker in patients with PTC (69,70). In a previous study, ROC curve analysis demonstrated that hsa-circ-047771 was a diagnostic biomarker for the differentiation of PTC tissues from adjacent normal tissues. Functional experiments also reported that hsa-circ-047771 targeted miR-522-3p/miR-153-5p, and that these miRNAs were upregulated in PTC tissues (12). It can thus be concluded from these data that the downregulation of hsa-circ-047771 results in PTC cell progression in a miR-522-3p/miR-153-5p-dependent manner.

hsa-circ-0137287. In a previous study RT-qPCR demonstrated that hsa-circ-0137287, located on chromosome 8: 92301363-92307931, was downregulated in PTC tissues and cell lines. Clinicopathological characteristics, including extra-thyroidal extension, $\mathrm{T}$ stage, lymph node metastasis, microcarcinoma and tumor size among patients with PTC, 
were primarily associated with hsa-circ-0137287 expression. ROC curve analysis also determined that hsa-circ-0137287 could be used as a diagnostic biomarker (71). In conclusion, these pieces of information indicate that hsa-circ-0137287 is downregulated in PTC; however, further studies are required to explore the downstream mechanisms.

\section{Conclusion}

Thyroid cancer is the most prevalent disorder of the endocrine system. PTC is the most common type of thyroid cancer, exhibiting an increase in incidence worldwide (72). It has recently been demonstrated that the dysregulation of certain circRNAs results in PTC cell progression or suppression (34). These circRNAs may function as tumor-promoters or tumor-suppressors and usually function by sponging different miRNAs. It has previously been demonstrated that circRNAs possess an annular structure and are thus known as a stable class of RNA molecules. Based on their unique characteristics, circRNAs are promising diagnostic and/or prognostic biomarkers in various types of cancer (73). circRNAs contain multiple miRNA binding sites, enabling them to sponge miRNAs and modulate downstream mechanisms by controlling miRNA expression (74).

In PTC cells, researchers have discovered that certain defined circRNAs target miRNAs and regulate cell proliferation, invasion and apoptosis. However, there are some circRNAs, including hsa-circ-007148 (12) and hsa-circ-0137287 (71), in PTC cells, for which the downstream mechanisms have yet to be distinguished. The overexpression of circRNAs in PTC [e.g., circBACH2 (40) and circFOXM1 (38)] function as tumor-promoters and downregulated ones [e.g., circITCH (67) and hsa-circ-100395 (34)] function as tumor-suppressors. These circRNAs, their identified target genes/proteins, and their biological functions are presented in Table I. Taken together, circRNAs with their distinctive features, are more stable in cells and can even cause drug resistance, thus suggesting that they may be used for distinguishing and treating malignancies.

\section{Acknowledgements}

Not applicable.

\section{Funding}

No funding was received.

\section{Availability of data and materials}

Not applicable.

\section{Authors' contributions}

DQ conceived and designed the study. NS and TU contributed to the writing of the manuscript, and were involved in the literature search. All authors have read and approved the final manuscript.

\section{Ethics approval and consent to participate}

Not applicable.

\section{Patient consent for publication}

Not applicable.

\section{Competing interests}

The authors declare that they have no competing interests.

\section{References}

1. Cancer Genome Atlas Research Network: Integrated genomic characterization of papillary thyroid carcinoma. Cell 159: 676-690, 2014.

2. La Vecchia C, Malvezzi M, Bosetti C, Garavello W, Bertuccio P, Levi F and Negri E: Thyroid cancer mortality and incidence: A global overview. Int J Cancer 136: 2187-2195, 2015.

3. Siegel RL, Miller KD, Fedewa SA, Ahnen DJ, Meester RGS, Barzi A and Jemal A: Colorectal cancer statistics, 2017. CA Cancer J Clin 67: 177-193, 2017.

4. Xing M: BRAF mutation in papillary thyroid cancer: Pathogenic role, molecular bases, and clinical implications. Endocr Rev 28: 742-762, 2007.

5. Sheu SY,Grabellus F,Schwertheim S, WormK,Broecker-Preuss M and Schmid KW: Differential miRNA expression profiles in variants of papillary thyroid carcinoma and encapsulated follicular thyroid tumours. Br J Cancer 102: 376-382, 2010.

6. Xu B, Shao Q, Xie K,Zhang Y,Dong T, Xia Y and Tang W: The long non-coding RNA ENST00000537266 and ENST00000426615 influence papillary thyroid cancer cell proliferation and motility. Cell Physiol Biochem 38: 368-378, 2016.

7. Saporito D, Brock P, Hampel H, Sipos J, Fernandez S, Liyanarachchi S, de la Chapelle A and Nagy R: Penetrance of a rare familial mutation predisposing to papillary thyroid cancer. Fam Cancer 17: 431-434, 2018

8. Clarke CA, Reynolds P, Oakley-Girvan I, Lee E, Lu Y, Yang J, Moy LM, Bernstein L and Horn-Ross PL: Indicators of microbial-rich environments and the development of papillary thyroid cancer in the California teachers study. Cancer Epidemiol 39: 548-553, 2015 .

9. Viola D, Materazzi G, Valerio L, Molinaro E, Agate L, Faviana P, Seccia V, Sensi E, Romei C, Piaggi P, et al: Prophylactic central compartment lymph node dissection in papillary thyroid carcinoma: Clinical implications derived from the first prospective randomized controlled single institution study. J Clin Endocrinol Metab 100: 1316-1324, 2015.

10. Haugen BR, Alexander EK, Bible KC, Doherty GM, Mandel SJ, Nikiforov YE, Pacini F, Randolph GW, Sawka AM, Schlumberger M, et al: 2015 American Thyroid Association management guidelines for adult patients with thyroid nodules and differentiated thyroid cancer: The American Thyroid Association guidelines task force on thyroid nodules and differentiated thyroid cancer. Thyroid 26: 1-133, 2016.

11. Yin Y, Hong S, Yu S, Huang Y, Chen S, Liu Y, Zhang Q, Li Y and Xiao H: miR-195 inhibits tumor growth and metastasis in papillary thyroid carcinoma cell lines by targeting CCND1 and FGF2. Int J Endocrinol 2017: 6180425, 2017.

12. Ren H, Liu Z, Liu S, Zhou X, Wang H, Xu J, Wang D and Yuan G: Profile and clinical implication of circular RNAs in human papillary thyroid carcinoma. PeerJ 6: e5363, 2018.

13. Xing $M$, Tufano RP, Tufaro AP, Basaria S, Ewertz M, Rosenbaum E, Byrne PJ, Wang J, Sidransky D and Ladenson PW: Detection of BRAF mutation on fine needle aspiration biopsy specimens: A new diagnostic tool for papillary thyroid cancer. J Clin Endocrinol Metab 89: 2867-2872, 2004.

14. Kimura ET, Nikiforova MN, Zhu Z, Knauf JA, Nikiforov YE and Fagin JA: High prevalence of BRAF mutations in thyroid cancer: Genetic evidence for constitutive activation of the RET/PTC-RAS-BRAF signaling pathway in papillary thyroid carcinoma. Cancer Res 63: 1454-1457, 2003.

15. Kwak JY, Kim EK, Chung WY, Moon HJ, Kim MJ and Choi JR: Association of BRAFV600E mutation with poor clinical prognostic factors and US features in Korean patients with papillary thyroid microcarcinoma. Radiology 253: 854-860, 2009.

16. Haugen BR: 2015 American Thyroid Association management guidelines for adult patients with thyroid nodules and differentiated thyroid cancer: What is new and what has changed? Cancer 123: 372-381, 2017. 
17. Vriens MR, Weng J, Suh I, Huynh N, Guerrero MA, Shen WT, Duh QY, Clark OH and Kebebew E: MicroRNA expression profiling is a potential diagnostic tool for thyroid cancer. Cancer 118: 3426-3432, 2012.

18. Chen LL and Yang L: Regulation of circRNA biogenesis. RNA Biol 12: 381-388, 2015.

19. Kos A, Dijkema R, Arnberg AC, van der Meide PH and Schellekens H: The hepatitis delta (delta) virus possesses a circular RNA. Nature 323: 558-560, 1986.

20. Conn VM, Hugouvieux V, Nayak A, Conos SA, Capovilla G, Cildir G, Jourdain A, Tergaonkar V, Schmid M, Zubieta C and Conn SJ: A circRNA from SEPALLATA3 regulates splicing of its cognate mRNA through R-loop formation. Nat Plants 3: $17053,2017$.

21. Chen Y, Li C, Tan C and Liu X: Circular RNAs: A new frontier in the study of human diseases. J Med Genet 53: 359-365, 2016.

22. Qian L, Yu S, Chen Z, Meng Z, Huang S and Wang P: The emerging role of circRNAs and their clinical significance in human cancers. Biochim Biophys Acta Rev Cancer 1870: 247-260, 2018

23. Hansen TB, Jensen TI, Clausen BH, Bramsen JB, Finsen B, Damgaard CK and Kjems J: Natural RNA circles function as efficient microRNA sponges. Nature 495: 384-388, 2013.

24. Li Y, Hu J, Li L, Cai S, Zhang H, Zhu X, Guan G and Dong X: Upregulated circular RNA circ_0016760 indicates unfavorable prognosis in NSCLC and promotes cell progression through miR-1287/GAGE1 axis. Biochem Biophys Res Commun 503. 2089-2094, 2018.

25. Memczak S, Jens M, Elefsinioti A, Torti F, Krueger J, Rybak A Maier L, Mackowiak SD, Gregersen LH, Munschauer M, et al: Circular RNAs are a large class of animal RNAs with regulatory potency. Nature 495: 333-338, 2013.

26. Soghli N, Qujeq D, Yousefi T and Soghli N: The regulatory functions of circular RNAs in osteosarcoma. Genomics S0888-7543 31052-31053, 2020.

27. Xu B, Yang T, Wang Z, Zhang Y, Liu S and Shen M: CircRNA CDR las/miR-7 signals promote tumor growth of osteosarcoma with a potential therapeutic and diagnostic value. Cancer Manag Res 10: 4871-4880, 2018

28. Kai D, Yannian L, Yitian C, Dinghao G, Xin Z and Wu J: Circular RNA HIPK3 promotes gallbladder cancer cell growth by sponging microRNA-124. Biochem Biophys Res Commun 503: 863-869, 2018

29. Ling H, Fabbri $M$ and Calin GA: MicroRNAs and other non-coding RNAs as targets for anticancer drug development Nat Rev Drug Discov 12: 847-865, 2013.

30. Huang H, Wei L, Qin T, Yang N, Li Z and Xu Z: Circular RNA ciRS-7 triggers the migration and invasion of esophageal squamous cell carcinoma via miR-7/KLF4 and NF- $\mathrm{KB}$ signals. Cancer Biol Ther 20: 73-80, 2019.

31. Arnaiz E, Sole C, Manterola L, Iparraguirre L, Otaegui D and Lawrie CH: CircRNAs and cancer: Biomarkers and master regulators. Semin Cancer Biol 58: 90-99, 2019.

32. Chen F, Feng Z, Zhu J, Liu P, Yang C, Huang R and Deng Z: Emerging roles of circRNA_NEK6 targeting miR-370-3p in the proliferation and invasion of thyroid cancer via Wnt signaling pathway. Cancer Biol Ther 19: 1139-1152, 2018.

33. Kulcheski FR, Christoff AP and Margis R: Circular RNAs are miRNA sponges and can be used as a new class of biomarker. J Biotechnol 238: 42-51, 2016.

34. Peng N, Shi L, Zhang Q, Hu Y, Wang N and Ye H: Microarray profiling of circular RNAs in human papillary thyroid carcinoma. PLoS One 12: e0170287, 2017.

35. Teng H, Mao F, Liang J, Xue M, Wei W, Li X, Zhang K, Feng D, Liu B and Sun Z: Transcriptomic signature associated with carcinogenesis and aggressiveness of papillary thyroid carcinoma. Theranostics 8: 4345-4358, 2018.

36. Griffith OL, Melck A, Jones SJ and Wiseman SM: Meta-analysis and meta-review of thyroid cancer gene expression profiling studies identifies important diagnostic biomarkers. J Clin Oncol 24: 5043-5051, 2006.

37. Yao Y, Chen X, Yang H, Chen W, Qian Y, Yan Z, Liao T, Yao W, Wu W, Yu T, et al: Hsa_circ_0058124 promotes papillary thyroid cancer tumorigenesis and invasiveness through the NOTCH3/GATAD2A axis. J Exp Clin Cancer Res 38: 318, 2019.

38. Pan Y, Xu T, Liu Y, Li W and Zhang W: Upregulated circular RNA circ 0025033 promotes papillary thyroid cancer cell proliferation and invasion via sponging miR-1231 and miR-1304. Biochem Biophys Res Commun 510: 334-338, 2019.
39. Yang Y, Ding L, Li Y and Xuan C: Hsa_circ_0039411 promotes tumorigenesis and progression of papillary thyroid cancer by miR-1179/ABCA9 and miR-1205/MTA1 signaling pathways. J Cell Physiol 235: 1321-1329, 2020.

40. Cai X, Zhao Z, Dong J, Lv Q, Yun B, Liu J, Shen Y, Kang J and Li J: Circular RNA circBACH2 plays a role in papillary thyroid carcinoma by sponging miR-139-5p and regulating LMO4 expression. Cell Death Dis 10: 184, 2019.

41. Zhang J, Zhang J, Qiu W, Zhang J, Li Y, Kong E, Lu A, Xu J and Lu X: MicroRNA-1231 exerts a tumor suppressor role through regulating the EGFR/PI3K/AKT axis in glioma. J Neurooncol 139: 547-562, 2018.

42. Wang H, Wu J, Luo W and Hu J: Low expression of miR-1231 in patients with glioma and its prognostic significance. Eur Rev Med Pharmacol Sci 22: 8399-8405, 2018.

43. Li CG, Pu MF, Li CZ, Gao M, Liu MX, Yu CZ, Yan H, Peng C, Zhao Y, Li Y, et al: MicroRNA-1304 suppresses human non-small cell lung cancer cell growth in vitro by targeting heme oxygenase-1. Acta Pharmacol Sin 38: 110-119, 2017.

44. Zhou GK, Zhang GY, Yuan ZN, Pei R and Liu DM: Has_circ_ 0008274 promotes cell proliferation and invasion involving AMPK/mTOR signaling pathway in papillary thyroid carcinoma. Eur Rev Med Pharmacol Sci 22: 8772-8780, 2018.

45. Cargnello M, Tcherkezian J and Roux PP: The expanding role of mTOR in cancer cell growth and proliferation. Mutagenesis 30: 169-176, 2015.

46. Liu F, Zhang J, Qin L, Yang Z, Xiong J, Zhang Y, Li R, Li S, Wang H, Yu B, et al: Circular RNA EIF6 (Hsa_circ_0060060) sponges miR-144-3p to promote the cisplatin-resistance of human thyroid carcinoma cells by autophagy regulation. Aging (Albany NY) 10: 3806-3820, 2018.

47. Xiao W, Lou N, Ruan H, Bao L, Xiong Z, Yuan C, Tong J, Xu G, Zhou Y, Qu Y, et al: Mir-144-3p promotes cell proliferation, metastasis, sunitinib resistance in clear cell renal cell carcinoma by downregulating ARID1A. Cellular Cell Physiol Biochem 43: 2420-2433, 2017

48. Zhao Y, Xie Z, Lin J and Liu P: MiR-144-3p inhibits cell proliferation and induces apoptosis in multiple myeloma by targeting c-Met. Am J Transl Res 9: 2437-2446, 2017.

49. Bi W, Huang J, Nie C, Liu B, He G, Han J,Pang R, Ding Z, Xu J and Zhang J: CircRNA circRNA_102171 promotes papillary thyroid cancer progression through modulating CTNNBIP1-dependent activation of $\beta$-catenin pathway. J Exp Clin Cancer Res 37: 275, 2018.

50. Fu X, Zhu X, Qin F, Zhang Y, Lin J, Ding Y, Yang Z, Shang Y, Wang L, Zhang Q and Gao Q: Linc00210 drives Wnt/ $\beta$-catenin signaling activation and liver tumor progression through CTNNBIP1-dependent manner. Mol Cancer 17: 73, 2018.

51. Li X, Tian Y, Hu Y, Yang Z, Zhang L and Luo J: CircNUP214 sponges miR-145 to promote the expression of ZEB2 in thyroid cancer cells. Biochem Biophys Res Commun 507: 168-172, 2018.

52. Liu Q, Chen J, Wang B, Zheng Y, Wan Y, Wang Y, Zhou L, Liu S, Li G and Yan Y: miR-145 modulates epithelial-mesenchymal transition and invasion by targeting ZEB2 in non-small cell lung cancer cell lines. J Cell Biochem: Dec 7, 2018 (Epub ahead of print).

53. Brown CY, Dayan S, Wong SW, Kaczmarek A, Hope CM, Pederson SM, Arnet V, Goodall GJ, Russell D, Sadlon TJ and Barry SC: FOXP3 and miR-155 cooperate to control the invasive potential of human breast cancer cells by down regulating ZEB2 independently of ZEB1. Oncotarget 9: 27708-27727, 2018.

54. Jin X, Wang Z, Pang W, Zhou J, Liang Y, Yang J, Yang L and Zhang Q: Upregulated hsa_circ_0004458 contributes to progression of papillary thyroid carcinoma by inhibition of miR-885-5p and activation of RAC1. Med Sci Monit 24: 5488-5500, 2018.

55. Chen QY, Zheng Y, Jiao DM, Chen FY, Hu HZ, Wu YQ, Song J Yan J, Wu LJ and Lv GY: Curcumin inhibits lung cancer cell migration and invasion through Racl-dependent signaling pathway. J Nutr Biochem 25: 177-185, 2014.

56. Becker MS, Müller PM, Bajorat J, Schroeder A, Giaisi M, Amin E, Ahmadian MR, Rocks O, Köhler R, Krammer PH and Li-Weber M: The anticancer phytochemical rocaglamide inhibits Rho GTPase activity and cancer cell migration. Oncotarget 7: 51908-51921, 2016

57. Ren S, Xin Z, Xu Y, Xu J and Wang G: Construction and analysis of circular RNA molecular regulatory networks in liver cancer. Cell Cycle 16: 2204-2211, 2017.

58. Liu T, Liu S, Xu Y, Shu R, Wang F, Chen C, Zeng Y and Luo H: Circular RNA-ZFR inhibited cell proliferation and promoted apoptosis in gastric cancer by sponging $\mathrm{miR}-130 \mathrm{a} / \mathrm{miR}-107$ and modulating PTEN. Cancer Res Treat 50: 1396-1417, 2018. 
59. Wei H, Pan L, Tao D and Li R: Circular RNA circZFR contributes to papillary thyroid cancer cell proliferation and invasion by sponging miR-1261 and facilitating C8orf4 expression. Biochem Biophys Res Commun 503: 56-61, 2018.

60. Sunde M, McGrath KC, Young L, Matthews JM, Chua EL, Mackay JP and Death AK: TC-1 is a novel tumorigenic and natively disordered protein associated with thyroid cancer. Cancer Res 64: 2766-2773, 2004.

61. Lei J, Li W, Yang Y, Lu Q, Zhang N, Bai G, Zhong D, Su K, Liu B, Li X, et al: TC-1 overexpression promotes cell proliferation in human non-small cell lung cancer that can be inhibited by PD173074. PLoS One 9: e100075, 2014.

62. Tao L, Yang L, Tian P, Guo X and Chen Y: Knockdown of circPVT1 inhibits progression of papillary thyroid carcinoma by sponging miR-126. RSC Adv 9: 13316-13324, 2019.

63. Kitano M, Rahbari R, Patterson EE, Xiong Y, Prasad NB, Wang Y, Zeiger MA and Kebebew E: Expression profiling of difficult-to-diagnose thyroid histologic subtypes shows distinct expression profiles and identify candidate diagnostic microRNAs. Ann Surg Oncol 18: 3443-34452, 2011.

64. Wu G, Zhou W, Lin X, Sun Y, Li J, Xu H, Shi P, Gao L and Tian X: CircRASSF2 Acts as ceRNA and promotes papillary thyroid carcinoma progression through miR-1178/TLR4 signaling pathway. Mol Ther Nucleic Acids 19: 1153-1163, 2020.

65. Zhang W, Zhang H and Zhao X: Circ_0005273 promotes thyroid carcinoma progression by SOX2 expression. Endocr Relat Cancer 27: 11-21, 2020.

66. Li F, Ma K, Sun M and Shi S: Identification of the tumor-suppressive function of circular RNA ITCH in glioma cells through sponging miR-214 and promoting linear ITCH expression. Am J Transl Res 10: 1373-1386, 2018.

67. Wang M, Chen B, Ru Z and Cong L: CircRNA circ-ITCH suppresses papillary thyroid cancer progression through miR-22-3p/CBL/ $\beta$-catenin pathway. Biochem Biophys Res Commun 504: 283-288, 2018.
68. Shashar M, Siwak J, Tapan U, Lee SY, Meyer RD, Parrack P, Tan J, Khatami F, Francis J, Zhao Q, et al: C-Cbl mediates the degradation of tumorigenic nuclear $\beta$-catenin contributing to the heterogeneity in Wnt activity in colorectal tumors. Oncotarget 7: 71136-71150, 2016.

69. Lupi C, Giannini R, Ugolini C, Proietti A, Berti P, Minuto M, Materazzi G, Elisei R, Santoro M, Miccoli P and Basolo F: Association of BRAF V600E mutation with poor clinicopathological outcomes in 500 consecutive cases of papillary thyroid carcinoma. J Clin Endocrinol Metab 92: 4085-4090, 2007.

70. Elisei R, Ugolini C, Viola D, Lupi C, Biagini A, Giannini R, Romei C, Miccoli P, Pinchera A and Basolo F: BRAF(V600E) mutation and outcome of patients with papillary thyroid carcinoma: A 15-year median follow-up study. J Clin Endocrinol Metab 93: 3943-3949, 2008.

71. Lan X, Cao J, Xu J, Chen C, Zheng C, Wang J, Zhu X, Zhu X and Ge M: Decreased expression of hsa_circ_0137287 predicts aggressive clinicopathologic characteristics in papillary thyroid carcinoma. J Clin Lab Anal 32: e22573, 2018.

72. Siegel RL, Miller KD and Jemal A: Cancer statistics 2018. CA Cancer J Clin 68: 7-30, 2018.

73. Lan X, Xu J, Chen C, Zheng C, Wang J, Cao J, Zhu X and Ge M: The landscape of circular RNA expression profiles in papillary thyroid carcinoma based on RNA sequencing. Cell Physiol Biochem 47: 1122-1132, 2018.

74. Liu Q, Pan LZ, Hu M and Ma JY: Molecular Network-Based identification of circular RNA-Associated ceRNA network in papillary thyroid cancer. Pathol Oncol Res 2019 [Epub ahead of print].

This work is licensed under a Creative Commons Attribution-NonCommercial-NoDerivatives 4.0 International (CC BY-NC-ND 4.0) License. 